\title{
Stability analysis of HIV-1 model with multiple delays
}

\author{
Nigar Ali , Gul Zaman ${ }^{1 *}$ and Obaid Algahtani
}

\author{
*Correspondence: \\ gzaman@uom.edu.pk \\ 'Department of Mathematics, \\ University of Malakand, Chakdara \\ Dir (Lower), Khyber Pukhtoonkhwa, \\ Pakistan \\ Full list of author information is \\ available at the end of the article
}

\begin{abstract}
A mathematical model for HIV-1 infection with multiple delays is proposed. These delays account for (i) the delay in contact process between the uninfected cells virus, (ii) a latent period between the time target cells which are contacted by the virus particles and the time the virions enter the cells, and (iii) a virus production period for new virions to be produced within and released from the infected cells. For this model, the basic reproductive number is identified and its threshold property is discussed. The uninfected and infected steady states are shown to be locally as well as globally asymptotically stable. The value of the basic reproductive number shows that increasing any one of these delays will decrease this number. This may suggest a new direction for new drugs that can prolong the infection process and spreading of virus. The proved results have potential applications in HIV-1 therapy.
\end{abstract}

\section{Introduction}

Mathematical modeling in epidemiology provides understanding of the mechanisms that influence the spread of a disease and suggests control strategies. Human immunodeficiency virus (HIV-1) is a lentivirus that causes acquired immunodeficiency syndrome (AIDS). The HIV infection is characterized by three different phases, namely, the primary infection, a clinically asymptomatic stage (chronic infection), and acquired immunodeficiency syndrome (AIDS). In recent years the population dynamics of infectious diseases have been extensively studied [1-19]. Clinical research combined with mathematical modeling has enhanced progress in the understanding of the HIV-1 infection [4]. This is because mathematical models can offer a way to study the dynamics of viral load in vivo and can be very useful in understanding the interaction between virus and host cell.

In the last decade, the HIV-infection models with time delays have been studied by many authors, and time delays of one type or another have been incorporated into biological models by many authors (e.g., [1-12, 14-19] and the references cited therein). The results presented in [17-19] have shown that larger intercellular delay may help eradicate the virus, while the activation of CTLs can only help reduce the virus load and increase the healthy $\mathrm{CD}+4$ cells in the long term sense. Pawelek et al. [7] have studied that models of HIV-1 infection incorporating intracellular delays are more accurate representations of the biology, and that they can change the estimated values of the kinetic parameters when compared to models without delays. Therefore, we incorporate time delay terms in the model in the interaction of different cells. 
This research extends the work in [16] by incorporating delays in the contact process between the uninfected cells and virus. By introducing these multiple delay terms, the proposed model becomes

$$
\begin{aligned}
\dot{x}(t) & =N-d x(t)-\beta e^{-m \tau_{1}} x\left(t-\tau_{1}\right) v\left(t-\tau_{1}\right), \\
\dot{y}(t) & =\beta e^{-m \tau_{1}} x\left(t-\tau_{1}\right) v\left(t-\tau_{1}\right)-a y(t)-p y(t) z(t), \\
\dot{v}(t) & =k y(t)-u v(t), \\
\dot{z}(t) & =c y\left(t-\tau_{2}\right) z\left(t-\tau_{2}\right)-b z(t),
\end{aligned}
$$

where $x(t), y(t)$, and $v(t)$ are the densities of uninfected target cells, infected target cells and free virus, respectively, at time $t$. $\beta$ is the infection rate of uninfected cells by the virus. The healthy cell is assumed to be produced at a constant rate $N$. It is also assumed that, once cells are infected, they may die at rate $a$ either due to the action of the virus or the immune system, in the meantime each producing HIV-1 virus particles at a rate $k$ during their life with an average length $1 / a$. The density $z$ represents CTL response cells. Here, $\tau_{1}$ can be regarded as the average time for a viral particle to go through the eclipse phase (or average latent period) and $\tau_{2}$ may be treated as the average time between the entry of a virion into a cell and the creation and release of new virions from this cell. Realistically, $\tau_{1}$ may differ from $\tau_{2} . e^{-m \tau_{1}}$ is the probability of surviving of cells in the time period from $\tau_{1}$ to $t$, where $m$ is assumed to be the constant death rate of infected CD4+ T cells. The novelty of the proposed model is that it considers a delay in the process of infection of healthy $\mathrm{T}$ cells, and in virus production. In the previous research the rate of contact of targeted cells and virus was ignored. This work considers the whole process of infection of healthy cells. In eliminating or controlling the disease after human body was infected by the virus, the immune response plays an important role. Antigenic stimulation in generating CTLs may need a period of time $\tau_{2}$, that is, the CTL response at time $t$ may depend on the population of antigen at a period time $\left(t-\tau_{2}\right)[20]$.

We study the dynamical behavior of the proposed model and show how delays influence the stability. We discuss the well-posedness of the solution of equilibria and their stability. In order to properly define biologically meaningful equilibria, we find the basic reproduction number. It will be shown that an infection-free equilibrium, $E_{0}$, is locally as well as globally asymptotically stable. We also show that the single-infection equilibrium, $E_{1}$, is locally as well as globally asymptotically stable, and the double-infection equilibrium, $E_{2}$, is also globally asymptotically stable.

The rest of this paper is organized as follows. The next section is devoted to the wellposedness and positivity of the solution. In Section 3, local and global stabilities of the infection-free equilibrium, $E_{0}$, are discussed, and in Section 4 we discuss the infectionfree equilibrium, $E_{1}$, and the double-infection equilibrium, $E_{2}$. A numerical simulation and conclusion are discussed in Section 5.

\section{Positivity and well-posedness of the solution and basic reproductive number}

In this section, first we discuss the positivity and well-posedness of the solution.

Theorem 2.1 All solutions of the system (1) remain non-negative, provided the given initial conditions are non-negative and bounded. 
Proof Let $X=C\left([-\tau, 0] ; R^{4}\right)$ be the Banach space of continuous mapping from $[-\tau, 0]$ to $R^{4}$ equipped with the sup-norm. It is biologically reasonable to consider the following initial conditions for the system (1):

$$
x(\phi) \geq 0, \quad y(\phi) \geq 0, \quad z(\phi) \geq 0, \quad v(\phi) \geq 0, \quad \phi \in[-\tau, 0],
$$

where $(x(\phi), y(\phi), v(\phi), z(\phi)) \in X$ and $\tau=\max \left\{\tau_{1}, \tau_{2}\right\}$. By the fundamental theory of functional differential equations (see, e.g., $[20,21]$ ), we know that there exists a unique solution $x(t), y(t), z(t)$, and $v(t)$ for the given initial conditions in (2). By using the constant of the variation formulas, we get the following solution of the system (1):

$$
\begin{aligned}
& x(t)=x(0) e^{-\int_{0}^{t}(d) d \zeta}+\int_{0}^{t}\left(N-x\left(t-\tau_{1}\right) v\left(t-\tau_{1}\right) \beta e^{-m \tau_{1}}\right) e^{-\int_{0}^{t}(d) d \zeta} d \eta, \\
& y(t)=y(0) e^{-\int_{0}^{t}(a+p z(\zeta)) d \zeta}+\int_{0}^{t}\left(\beta e^{-m \tau_{1}} x\left(t-\tau_{1}\right) \nu\left(t-\tau_{1}\right)\right) e^{-\int_{\eta}^{t}(a+p v(\zeta)) d \zeta} d \eta, \\
& z(t)=z(0) e^{-b t}+\int_{0}^{t} c y\left(t-\tau_{2}\right) z\left(t-\tau_{2}\right) e^{-b(t-\eta)} d \eta, \\
& v(t)=v(0) e^{-u t}+\int_{0}^{t} k y(\eta) e^{-u(t-\eta)} d \eta,
\end{aligned}
$$

which clearly indicates that all the solutions are positive. In order to show the boundedness of the solution $x(t), y(t), z(t)$, and $v(t)$, we define

$$
B(t)=x(t)+y(t)+\frac{a}{2 k} v(t)+\frac{p}{c} z\left(t+\tau_{2}\right)
$$

Calculating the derivative of equation (3) and using system (1) yield

$$
\begin{aligned}
\frac{d B(t)}{d t}= & N-d x(t)-\beta e^{-m \tau_{1}} x\left(t-\tau_{1}\right) v\left(t-\tau_{1}\right)+\beta e^{-m \tau_{1}} x\left(t-\tau_{1}\right) v\left(t-\tau_{1}\right) \\
& -a y(t)-p y(t) z(t)+\frac{a}{2 k}(k y(t)-u v(t))+\frac{b k}{2}(c z(t)-q w(t)) \\
= & N-\left(d x(t)+\frac{a}{2} y(t)+u \frac{a}{2 k} v(t)+b \frac{p}{c} z\left(t+\tau_{2}\right)\right) \\
\leq & N-\delta B(t) .
\end{aligned}
$$

Here $\delta=\min \left(d, \frac{a}{2}, u, b\right)$. This implies that $B(t)$ is bounded for large $t$. So $x(t), y(t), v(t)$, and $z(t)$ are ultimately bounded.

Now we discuss the equilibria of the system (1) which has three possible biological meaningful equilibria. We have the disease-free equilibrium, $E_{0}\left(x_{0}, y_{0}, v_{0}, z_{0}\right)$, the singleinfection equilibrium, $E_{1}\left(x_{1}, y_{1}, v_{1}, z_{1}\right)$, and the double-infection equilibrium, $E_{2}\left(x_{2}, y_{2}\right.$, $\left.v_{2}, z_{2}\right)$, which are given by

$$
\begin{aligned}
& E_{0}=\left(\frac{N}{d}, 0,0,0,0\right), \\
& E_{1}=\left(\frac{a u}{\beta k e^{-m \tau_{1}}}, \frac{u d\left(R_{0}-1\right)}{\beta k e^{-m \tau_{1}}}, \frac{d\left(R_{0}-1\right)}{\beta e^{-m \tau_{1}}}, 0\right),
\end{aligned}
$$




$$
E_{2}=\left(\frac{N u c}{\beta k b e^{-m \tau_{1}}+u c d}, \frac{b}{c}, \frac{b k}{u c}, \frac{\beta k N c a e^{-m \tau_{1}}-a\left(c d u+b k \beta e^{-m \tau_{1}}\right)}{p\left(c d u+k b \beta e^{-m \tau_{1}}\right)}\right) .
$$

From the biological meaning of the basic reproduction number (see [16]), we define

$$
R_{0}=\frac{k \beta N e^{-m \tau_{1}}}{a d u}
$$

where $\frac{N}{d}$ is the average number of healthy cells available for infection, $\frac{\beta e^{-m \tau_{1}}}{a}$ is the average number of host cells that each HIV-1 virus infects and $\frac{k}{u}$ is the average number of virons that an infected cell produces. If $R_{0}<1$, then $E_{0}$ is the only biologically meaningful equilibrium. If $R_{0}>1$, there are other biologically meaningful equilibria, $E_{1}$ and $E_{2}$.

\section{Stability of the disease-free equilibrium $E_{0}$}

In this section, we show the dynamical behavior of the system (1) at $E_{0}$.

Theorem 3.1 When $R_{0}<1$, then the disease-free equilibrium $E_{0}$ is locally asymptotically stable while for $R_{0}>1, E_{0}$ becomes unstable and the single-infection equilibrium $E_{1}$ occurs.

Proof The characteristic equation of the Jacobian matrix corresponding to the linearized system (1) at $E_{0}$ is given by

$$
\operatorname{det}\left[\gamma I-J\left(E_{0}\right)\right]=\operatorname{det}\left(\begin{array}{cccc}
\gamma+d & 0 & \beta x_{0} e^{-\tau_{1}(\gamma+m)} & 0 \\
0 & \gamma+a & -\beta x_{0} e^{-\tau_{1}(\gamma+m)} & p \\
0 & -k & \gamma+u & 0 \\
0 & 0 & 0 & \gamma+b
\end{array}\right)=0
$$

where $J\left(E_{0}\right)$ denotes the Jacobian matrix at $E_{0}$.

After some simplification, equation (4) takes the form

$$
\operatorname{det}\left[\gamma I-J\left(E_{0}\right)\right]=(b+\gamma)(d+\gamma)\left[(a+\gamma)(u+\gamma)-\frac{N}{d} \beta k e^{-\tau_{1}(\gamma+m)}\right]=0 .
$$

The two roots of the characteristic equation (5) are $\gamma_{1}=-b$ and $\gamma_{2}=-d$ and the remaining roots can be obtained from the following equation:

$$
(a+\gamma)(u+\gamma)=\frac{N}{d} \beta k e^{-\tau_{1}(\gamma+m)} .
$$

If $\gamma$ has a non-negative real part then the modulus of the left-hand side of equation (6) satisfies

$$
|(a+\gamma)(u+\gamma)| \geq a u .
$$

The modulus of the right-hand side of equation (6) satisfies

$$
\frac{N}{d} \beta k\left|e^{-\tau_{1}(\gamma+m)}\right|=\left|a u R_{0}\right|<a u,
$$


which is contradiction. Hence, the real part of $\gamma$ has no non-negative part and the infection-free state $E_{0}$ is locally asymptotically stable when $R_{0}<1$. For $R_{0}>1$, we have

$$
\begin{aligned}
h(\gamma) & =(a+\gamma)(u+\gamma)-\frac{N}{d} \beta k e^{-\tau_{1}(\gamma+m)} \\
& =\gamma^{2}+(u+a) \gamma+a u\left(1-e^{-\gamma \tau_{1}} R_{0}\right) .
\end{aligned}
$$

Hence $h(0)=a u\left(1-R_{0}\right)<0$ and $\lim _{\gamma \rightarrow \infty} h(\gamma)=+\infty$. By the continuity of $h(\eta)$, there exists at least one positive root of $h(\gamma)=0$. Thus, the infection-free equilibrium, $E_{0}$, is unstable if $R_{0}>1$ (see [16]).

Theorem 3.2 If $R_{0}<1$, then the disease-free equilibrium $E_{0}$ is globally asymptotically stable.

Proof Let us consider the following Lyapunov functional:

$$
\begin{aligned}
L_{0}(t)= & x_{0}\left(\frac{x}{x_{0}}-\ln \left(\frac{x}{x_{0}}\right)-1\right)+y(t)+\frac{a}{k} v(t)+\frac{p}{c} z(t) \\
& +x_{0} \beta e^{-m \tau_{1}} \int_{t-\tau_{1}}^{t} \frac{x(\zeta) v(\zeta) d(\zeta)}{x\left(\zeta+\tau_{1}\right)} \\
& +p \int_{t-\tau_{2}}^{t} y(\zeta) z(\zeta) d(\zeta) .
\end{aligned}
$$

By taking the derivative of equation (7) and by using the system (1), we have

$$
\begin{aligned}
\dot{L}_{0}(t)= & \left(1-\frac{x_{0}}{x}\right)\left(N-d x(t)-\beta e^{-m \tau_{1}} x\left(t-\tau_{1}\right) v\left(t-\tau_{1}\right)\right)+\left(\beta x_{0} e^{-m \tau_{1}} y\left(t-\tau_{1}\right) z\left(t-\tau_{1}\right)\right. \\
& -a y(t)-p y(t) z(t))+\frac{a}{k}(k y(t)-u v(t))+\frac{p}{c}\left(c y\left(t-\tau_{2}\right) z\left(t-\tau_{2}\right)-b z(t)\right) \\
& +\beta x_{0} e^{-m \tau_{1}}\left(\frac{x(t) v(t)}{x\left(t+\tau_{1}\right)}-\frac{x\left(t-\tau_{1}\right) v\left(t-\tau_{1}\right)}{x(t)}\right) \\
& +p\left(y(t) z(t)-y\left(t-\tau_{2}\right) z\left(t-\tau_{2}\right)\right) .
\end{aligned}
$$

After some simplification equation (8) yields

$$
\dot{L}_{0}(t)=-\frac{d}{x}\left(x(t)-x_{0}\right)^{2}+\left(\beta \frac{N}{d} e^{-m \tau_{1}} \frac{x(t)}{x\left(t+\tau_{1}\right)}-\frac{a u}{k}\right) v(t)-\frac{b p}{c} z(t) \leq 0 .
$$

If $\tau_{1}$ is very large, then the rate of infection is very small and on the other hand, if $\tau_{1}$ is very small, then the infection will spread more rapidly so we take $x(t)=x\left(t+\tau_{1}\right)$. Therefore, the above equation (9) becomes

$$
\dot{L}_{0}(t)=-\frac{d}{x}\left(x(t)-x_{0}\right)^{2}-\frac{a u}{k}\left(1-R_{0}\right) v(t)-\frac{b p}{c} z(t) .
$$

Thus, if $R_{0}<1$, then equation (10) implies that $\dot{L}_{0}(t)<0$ and the equality holds only when $x_{0}=\frac{N}{d}, y(t)=0, z(t)=0, v(t)=0, w(t)=0$. Therefore, by LaSalle's invariance principle (see [22]), we conclude that $E_{0}$ is globally asymptotically stable when $R_{0}<1$. 


\section{Stability of single- and double-infection equilibria}

In this section, we discuss the single-infection-free equilibrium, $E_{1}$.

Theorem 4.1 $E_{1}$ is locally asymptotically stable if $1<R_{0}<1+\frac{b \beta k^{-m \tau_{1}}}{c d u}$, provided that $\tau_{1}, \tau_{2} \geq 0$, otherwise $E_{1}$ is unstable.

Proof The characteristic equation of the Jacobian matrix corresponding to the linearized system (1) at $E_{0}$ is given by

$$
\begin{aligned}
& \operatorname{det}\left[\gamma I-J\left(E_{1}\right)\right] \\
& =\operatorname{det}\left(\begin{array}{cccc}
\gamma+d+\beta v_{1} e^{-\tau_{1}(m+\gamma)} & 0 & \beta x_{1} e^{-\tau_{1}(\gamma+m)} & 0 \\
-\beta v_{1} e^{-\tau_{1}(m+\gamma)} & \gamma+a & -\beta x_{1} e^{-\tau_{1}(\gamma+m)} & p \\
0 & -k & \gamma+u & 0 \\
0 & 0 & 0 & \gamma+b-c y_{1} e^{-\gamma \tau_{2}}
\end{array}\right)=0 .
\end{aligned}
$$

After some fundamental calculation, we get the above characteristic equation in the following form:

$$
\left(\gamma+b-c y_{1} e^{-\gamma \tau_{2}}\right)\left[\gamma^{3}+a_{0} \gamma^{2}+a_{1} \gamma+a_{2}+\left(b_{0} \gamma^{2}+b_{1} \gamma+b_{2}\right) e^{-\tau_{1} \gamma}\right]=0,
$$

where

$$
\begin{array}{ll}
a_{0}=a+u+d, & a_{1}=(a+u) d+a u, \quad a_{2}=a u d, \\
b_{0}=\beta v_{1} e^{-m \tau_{1}}, & b_{1}=(a+u) \beta v_{1} e^{-m \tau_{1}}-k \beta x_{1} e^{-m \tau_{1}}, \quad b_{2}=a u \beta v_{1} e^{-m \tau_{1}} .
\end{array}
$$

First, we discuss the following factor of equation (11):

$$
\gamma^{3}+a_{0} \gamma^{2}+a_{1} \gamma+a_{2}+\left(b_{0} \gamma^{2}+b_{1} \gamma+b_{2}\right) e^{-\tau_{1} \gamma}=0 .
$$

Now, we present possible cases of delay term $\tau_{1}$. When $\tau_{1}=0$, then equation (12) becomes

$$
\gamma^{3}+c_{0} \gamma^{2}+c_{1} \gamma+c_{2}=0
$$

where

$$
\begin{aligned}
& c_{0}=a_{0}+b_{0}=a+u+d+d\left(R_{0}-1\right)>0, \\
& c_{1}=a_{1}+b_{1}=(a+u) d+(a+u) d\left(R_{0}-1\right)>0, \\
& c_{2}=a_{2}+b_{2}=a u d+a u d\left(R_{0}-1\right)>0, \\
& c_{1}-c_{0} c_{2}=d R_{0}\left(a^{2}+(a+u)\left(u+d R_{0}\right)\right)>c_{2}=a u d+\operatorname{aud}\left(R_{0}-1\right) .
\end{aligned}
$$

Thus, by using the Routh-Hurtwitz criterion in [23] one has no positive roots when $\tau_{1}=0$. Next, we consider the root distribution of equation (12) when $\tau_{1} \neq 0$. If $i \kappa(\kappa>0)$ is a solution of equation (12), then, separating real and imaginary parts, we get the following equations:

$$
\begin{aligned}
& a_{1} \kappa-\kappa^{3}=\left(b_{2}-b_{0} \kappa^{2}\right) \sin \kappa \tau_{1}-b_{1} \kappa \cos \kappa \tau_{1}, \\
& a_{0} \kappa^{2}-a_{2}=\left(b_{2}-b_{0} \kappa^{2}\right) \cos \kappa \tau_{1}+b_{1} \kappa \sin \kappa \tau .
\end{aligned}
$$


By squaring and adding the above equations, we get

$$
\kappa^{6}+m_{1} \kappa^{4}+m_{2} \kappa^{2}+m_{3}=0,
$$

where

$$
\begin{aligned}
& m_{1}=a_{0}^{2}-2 a_{1}-b_{0}^{2}, \\
& m_{2}=a_{1}^{2}-2 a_{0} a_{2}+2 b_{0} b_{2}-b_{1}^{2}, \\
& m_{3}=a_{2}^{2}-b_{2}^{2} .
\end{aligned}
$$

Let us suppose that $\sigma=\kappa^{2}>0$, then equation (14) becomes

$$
\sigma^{3}+m_{1} \sigma^{2}+m_{2} \sigma+m_{3}=0
$$

where

$$
\begin{aligned}
m_{1} & =a_{0}^{2}-2 a_{1}-b_{0}^{2}=a^{2}+u^{2}+d^{2}-\left(R_{0}-1\right)^{2} d^{2}>a^{2}+u^{2}, \\
m_{2} & =a_{1}^{2}-2 a_{0} a_{2}+2 b_{0} b_{2}-b_{1}^{2} \\
& =(a d)^{2}+(u d)^{2}+2 a u\left(d\left(R_{0}-1\right)\right)^{2}+(a+u) d\left(R_{0}-1\right)\left(2 a u-(a+u) d\left(R_{0}-1\right)\right) \\
& >(a d)^{2}+(u d)^{2}+2 a u(a+u) d\left(R_{0}-1\right), \\
m_{3} & =(a u d)^{2}-\left(a u d\left(R_{0}-1\right)^{2}\right)>(a u d)^{2}\left(R_{0}-1\right) .
\end{aligned}
$$

Hence, if $R_{0}>1$, then equation (15) has no positive roots. It is to be noted that the equilibrium $E_{1}$ is locally asymptotically stable by the general theory on characteristic equations of delay differential equations [23].

To find the other root, we consider the second factor of equation (11) to be given by

$$
\gamma+b-c y_{1} e^{-\gamma \tau_{2}}=0
$$

If $\tau_{2}=0$, then for $1<R_{0}<1+\frac{b \beta k e^{-m \tau_{1}}}{c d u}$, we have

$$
\gamma=c y_{1}-b=\frac{c}{k \beta e^{-m \tau_{1}}}\left(R_{0}-\left(1+\frac{b \beta k e^{-m \tau_{1}}}{c d u}\right)\right)<0 .
$$

This shows that the root of equation (16) is negative for $\tau_{2}=0$. To discuss the roots in the case $\tau_{2}>0$, we assume $\gamma=\kappa i(\kappa>0)$ to be a pure imaginary root of equation (16), to get

$$
\begin{aligned}
& \kappa=c\left(\frac{\beta N k e^{-m \tau_{1}}-a u d}{a \beta k e^{-m \tau_{1}}}\right) \sin \kappa \tau_{2}, \\
& b=c\left(\frac{\beta N k e^{-m \tau_{1}}-a u d}{a \beta k e^{-m \tau_{1}}}\right) \cos \kappa \tau_{2},
\end{aligned}
$$

which implies that

$$
\kappa^{2}=\left[c\left(\frac{\beta N k e^{-m \tau_{1}}-a u d}{a \beta k e^{-m \tau_{1}}}\right)\right]^{2}-b^{2} .
$$

However, for $1<R_{0}<1+\frac{b \beta k e^{-m \tau_{1}}}{c d u}$ this implies that $\kappa^{2}<0$, which is a contradiction. 
Thus, we conclude that all the roots of equation (16) have a negative real part when $\tau_{2} \geq 0$. Therefore, the equilibrium $E_{1}$ is locally asymptotically stable, when $1<R_{0}<1+$ $\frac{b \beta k e^{-m \tau_{1}}}{c d u}$ and $\tau_{1}, \tau_{2} \geq 0$.

Theorem 4.2 The single-infection-free equilibrium, $E_{1}$, is globally asymptotically stable, if $1<R_{0}<1+\frac{b \beta k e^{-m \tau_{1}}}{c d u}$, while for $R_{0}>1+\frac{b \beta k e^{-m \tau_{1}}}{c d u}, E_{1}$ is unstable.

Proof Denote $f(\rho)=\rho-1-\ln \rho, \rho \in R^{+}$. Let us construct the Lyapunov functional

$$
\begin{aligned}
L_{1}(t)= & x_{1} f\left(\frac{x}{x_{1}}\right)+y_{1} f\left(\frac{x}{y_{1}}\right)+\frac{a}{k} v_{1} f\left(\frac{v}{v_{1}}\right)+\frac{p}{c} z_{1} f\left(\frac{z}{z_{1}}\right) \\
& +\beta x_{1} v_{1} e^{-m \tau_{1}} \int_{t-\tau_{1}}^{t} f\left(\frac{x(\mu) v(\mu)}{x\left(\tau_{1}+\mu\right) v_{1}}\right) d \mu+p \int_{t-\tau_{2}}^{t} y(\mu) z(\mu) d \mu .
\end{aligned}
$$

By taking the derivative of the equation (17), we obtain

$$
\begin{aligned}
\dot{L}_{1}(t)= & \left(1-\frac{x_{1}}{x}\right)\left(\lambda-d x(t)-\beta e^{-m \tau_{1}} x\left(t-\tau_{1}\right) v\left(t-\tau_{1}\right)\right) \\
& +\left(1-\frac{y_{1}}{y}\right)\left(\beta e^{-m \tau_{1}} x\left(t-\tau_{1}\right) v\left(t-\tau_{1}\right)-a y(t)-p y(t) z(t)\right) \\
& +\frac{p}{c}\left(1-\frac{z}{z_{1}}\right)\left(c y\left(t-\tau_{2}\right) z\left(t-\tau_{2}\right)-b z(t)\right)+\frac{a}{k}\left(1-\frac{v_{1}}{v}\right)(k y(t)-u v(t)) \\
& +\beta x_{1} e^{-m \tau_{1}} \frac{x(t) v(t)}{x\left(\tau_{1}+t\right)}-\beta x_{1} e^{-m \tau_{1}} v_{1} \ln \left(\frac{x(t) v(t)}{x\left(\tau_{1}+t\right) \nu_{1}}\right) \\
& -\beta x_{1} e^{-m \tau_{1}} \frac{x\left(t-\tau_{1}\right) v\left(t-\tau_{1}\right)}{x\left(\tau_{1}+t\right)}+\beta x_{1} e^{-m \tau_{1}} v_{1} \ln \left(\frac{x\left(t-\tau_{1}\right) v\left(t-\tau_{1}\right)}{x\left(\tau_{1}+t\right) \nu_{1}}\right) .
\end{aligned}
$$

Using $E_{1}$ in the system (1) yields the following identities:

$$
\begin{aligned}
& \lambda=d x_{1}+\beta x_{1} v_{1} e^{-m \tau_{1}}, \\
& a y_{1}=\beta e^{-m \tau_{1}} x_{1} v_{1}, \\
& k y_{1}=u v_{1} .
\end{aligned}
$$

Using the above identities in equation (18), we get

$$
\begin{aligned}
\dot{L}_{1}(t)= & e^{-m \tau_{1}}\left(2-\frac{x_{1}}{x}-\frac{x}{x_{1}}\right)+\beta x_{1} v_{1} e^{-m \tau_{1}}\left(3-\frac{x_{1}}{x}-\frac{y v_{1}}{y_{1} v}-\frac{y_{1} x\left(t-\tau_{1}\right) v\left(t-\tau_{1}\right)}{x_{1} v_{1} y}\right. \\
& \left.-\ln \left(\frac{x(t) v(t)}{x\left(t-\tau_{1}\right) v\left(t-\tau_{1}\right)}\right)\right)+\frac{b p}{c}\left(R_{0}-\left(1+\frac{b \beta k e^{-m \tau_{1}}}{c d u}\right)\right) z \\
& +\left(\frac{a u}{k} \frac{x(t)}{x\left(t+\tau_{1}\right)}-\frac{a u}{k}\right) v .
\end{aligned}
$$

When $\tau_{1}$ is very large, then $x(t)=x\left(t+\tau_{1}\right)$. Thus, the above equation (19) can be written as

$$
\begin{aligned}
\dot{L}_{1}(t)= & e^{-m \tau_{1}}\left(2-\frac{x_{1}}{x}-\frac{x}{x_{1}}\right)+\beta x_{1} v_{1} e^{-m \tau_{1}}\left(3-\frac{x_{1}}{x}-\frac{y v_{1}}{y_{1} v}-\frac{y_{1} x\left(t-\tau_{1}\right) v\left(t-\tau_{1}\right)}{x_{1} v_{1} y}\right. \\
& \left.-\ln \left(\frac{x(t) v(t)}{x\left(t-\tau_{1}\right) v\left(t-\tau_{1}\right)}\right)\right)+\frac{b p}{c}\left(R_{0}-\left(1+\frac{b \beta k e^{-m \tau_{1}}}{c d u}\right)\right) z .
\end{aligned}
$$


To show that $\dot{L}_{1}(t)<0$, we need to prove that the following inequalities hold:

$$
\begin{aligned}
& e^{-m \tau_{1}}\left(2-\frac{x_{1}}{x}-\frac{x}{x_{1}}\right) \leq 0, \\
& \left(3-\frac{x_{1}}{x}-\frac{y v_{1}}{y_{1} v}-\frac{y_{1} x\left(t-\tau_{1}\right) v\left(t-\tau_{1}\right)}{x_{1} v_{1} y}-\ln \left(\frac{x t(t) v(t)}{x\left(t-\tau_{1}\right) v\left(t-\tau_{1}\right)}\right)\right) \leq 0 .
\end{aligned}
$$

Thus, the above results are satisfied only if $R_{0}<1+\frac{b \beta k e^{-m \tau_{1}}}{c d u}$, then equation (21) implies that $\frac{d L_{1}}{d t} \leq 0$. Moreover, the equality holds when $x=x_{1}$ and $y=y_{1}, v=v_{1}$, and $z=z_{1}$. Thus, by LaSalle's invariance principle [22], we conclude that $E_{1}$ is globally asymptotically stable.

Theorem 4.3 If $\tau_{1} \neq 0$ and $\tau_{2} \neq 0$ and $R_{0}>1+\frac{b \beta k e^{-m \tau_{1}}}{c d u}$, then the double-infection equilibrium, $E_{2}$, is globally asymptotically stable.

Proof Let us construct the Lyapunov functional given by

$$
\begin{aligned}
L_{2}(t)= & x_{2} f\left(\frac{x}{x_{2}}\right)+y_{1} f\left(\frac{x}{y_{2}}\right)+\frac{a}{k} \nu_{2} f\left(\frac{v}{v_{2}}\right)+\frac{p}{c} z_{2} f\left(\frac{z}{z_{2}}\right) \\
& +\beta x_{2} \nu_{2} e^{-m \tau_{1}} \int_{t-\tau_{1}}^{t} f\left(\frac{x(\mu) \nu(\mu)}{x\left(\tau_{1}+\mu\right) \nu_{2}}\right) d \mu+p \int_{t-\tau_{2}}^{t} y(\mu) z(\mu) d \mu .
\end{aligned}
$$

Using $E_{2}$ in the system (1), we get the following identities:

$$
\begin{aligned}
& N-d x_{2}=\beta x_{2} v_{2} e^{-m \tau_{1}}, \\
& \beta x_{2} v_{2} e^{-m \tau_{1}}=a y_{2}+p y_{2} z_{2}, \\
& k y_{2}=u v_{2}, \\
& b y_{2}=c .
\end{aligned}
$$

By taking the derivative of equation (22) and using the above identities, we get

$$
\begin{aligned}
\dot{L}_{2}(t)= & e^{-m \tau_{1}}\left(2-\frac{x_{2}}{x}-\frac{x}{x_{2}}\right)+\beta x_{2} v_{1} e^{-m \tau_{1}}\left(3-\frac{x_{2}}{x}-\frac{y v_{2}}{y_{2} v}-\frac{y_{2} x\left(t-\tau_{1}\right) v\left(t-\tau_{1}\right)}{x_{2} v_{2} y}\right. \\
& \left.+\ln \left(\frac{x\left(t-\tau_{1}\right) v\left(t-\tau_{1}\right)}{x(t) v(t)}\right)\right)+\left(\beta x_{2} e^{-m \tau_{1}} \frac{x(t)}{x\left(t+\tau_{1}\right)}-\frac{a u}{k}\right) v \\
& -p y_{2} z_{2}-p y z_{2}+p z_{2} v_{2} \frac{y}{v}+p y_{2} z_{2} \frac{v}{v_{2}} .
\end{aligned}
$$

When $\tau_{1}$ is very large and $x(t)=x\left(t+\tau_{1}\right)$ then equation (23) becomes

$$
\begin{aligned}
\dot{L}_{2}(t)= & e^{-m \tau_{1}}\left(2-\frac{x_{2}}{x}-\frac{x}{x_{2}}\right)+\beta x_{2} v_{2} e^{-m \tau_{1}}\left(3-\frac{x_{2}}{x}-\frac{y v_{2}}{y_{2} v}-\frac{y_{2} x\left(t-\tau_{1}\right) v\left(t-\tau_{1}\right)}{x_{2} v_{2} y}\right. \\
& \left.-\ln \left(\frac{x(t) v(t)}{x\left(t-\tau_{1}\right) v\left(t-\tau_{1}\right)}\right)\right)-\frac{p}{a c d u}\left(R_{0}-\left(1+\frac{b \beta k e^{-m \tau_{1}}}{c d u}\right)\right)\left(\left(\frac{v_{2}}{v}-1\right) y\right. \\
& \left.-\left(\frac{v}{v_{2}}-1\right) y_{2}\right) .
\end{aligned}
$$


The following inequalities hold:

$$
\begin{aligned}
& e^{-m \tau_{1}}\left(2-\frac{x_{1}}{x}-\frac{x}{x_{1}}\right) \leq 0, \\
& \left(3-\frac{x_{1}}{x}-\frac{y v_{1}}{y_{1} v}-\frac{y_{1} x\left(t-\tau_{1}\right) v\left(t-\tau_{1}\right)}{x_{1} v_{1} y}-\ln \left(\frac{x t(t) v(t)}{x\left(t-\tau_{1}\right) v\left(t-\tau_{1}\right)}\right)\right) \leq 0 .
\end{aligned}
$$

Therefore, equation (24) implies that $\frac{d L_{2}}{d t} \leq 0$, when $R_{0}<1+\frac{b \beta k e^{-m \tau_{1}}}{a u c}$. Moreover, the equality holds when $x=x_{2}$ and $y=y_{2}, v=v_{2}$, and $z=z_{2}$. Thus by LaSalle's invariance principle [22], we conclude that $E_{2}$ is globally asymptotically stable.

\section{Numerical simulation and discussion}

In the previous sections, we studied dynamical behaviors of the system (1) and obtained some important results. For a numerical simulation of the proposed model, we used the parameter values given in Table 1.

Figure 1 represents the dynamical behavior of the densities of uninfected target cells $x(t)$, the infected target cells $y(t)$, the free virus $v(t)$, and the density $z$ represents the CTL response cells at time $t$. Our results show that by incorporating the delay term in the model one increases the number of CDT4 positive cells and decreases the uninfected cells.

In this paper we discuss a HIV-infection model by introducing delay terms in different interaction terms. Dynamical analysis of the system (1) shows that delays play an important role in the stability of the equilibrium. The detailed analytic study has shown that the extended model with delays, like the model with no delay, also has three equilibrium solutions. The disease-free equilibrium $E_{0}$, the single-infection equilibrium, $E_{1}$, and the double-infection equilibrium, $E_{2}$, and a series of bifurcations occur as the basic reproduction number is increased. One has shown that $E_{0}$ is globally asymptotically stable for $R_{0} \in(0,1)$ and becomes unstable at the transcritical bifurcation point $R_{0}=1$ and bifurcates into $E_{1}$, which is globally asymptotically stable for $R_{0}>1$. However, it loses it stability at another bifurcation point $R_{0}>1+\frac{b \beta k e^{-m \tau_{1}}}{c d u}$ and $E_{2}$ occurs. Also, it has been shown that $E_{2}$ is globally asymptotically stable.

From the reproductive number $R_{0}\left(\tau_{1}\right)=\frac{k \beta N e^{-m \tau_{1}}}{a d p}$, which is a function of $\tau_{1}$, we see that it is decreasing in delay $\tau_{1}$ with $R_{0}(\infty)=0$, which means that the intracellular delay $\tau_{1}$ plays a positive role in preventing the virus. Because the larger $\tau_{1}$ can bring $R_{0}$ to a level lower than one. When the delay is chosen as the bifurcation parameter, it is shown that the delay plays an important role in determining the dynamical behavior of the system.

Table 1 Parameters used for numerical simulation

\begin{tabular}{llll}
\hline Notation & Parameter definition & Value & Source \\
\hline$N$ & recruitment rate & 160 & {$[20]$} \\
$d$ & death rate of uninfected target cells & 0.16 & assumed \\
$P$ & infection rate of uninfected cells by virus & 0.002 & {$[21]$} \\
$a$ & death rate of productively infected cells & 1.85 & {$[20]$} \\
$P$ & killing rate of infected cells by CTL response cells & 0.2 & assumed \\
$k$ & rate of the virus particles produced by infected cells & 1,200 & {$[20]$} \\
$u$ & viral clearance rate constant & 8 & assumed \\
$C$ & rate at which the CTL response is produced & 0.2 & {$[21]$} \\
$b$ & death rate of the CTL response & 0.4 & assumed \\
$T 1$ & intracellular delay & 0.2 & {$[21]$} \\
$T 2$ & delay in antigenic stimulation & 2.4 & {$[21]$} \\
\hline
\end{tabular}




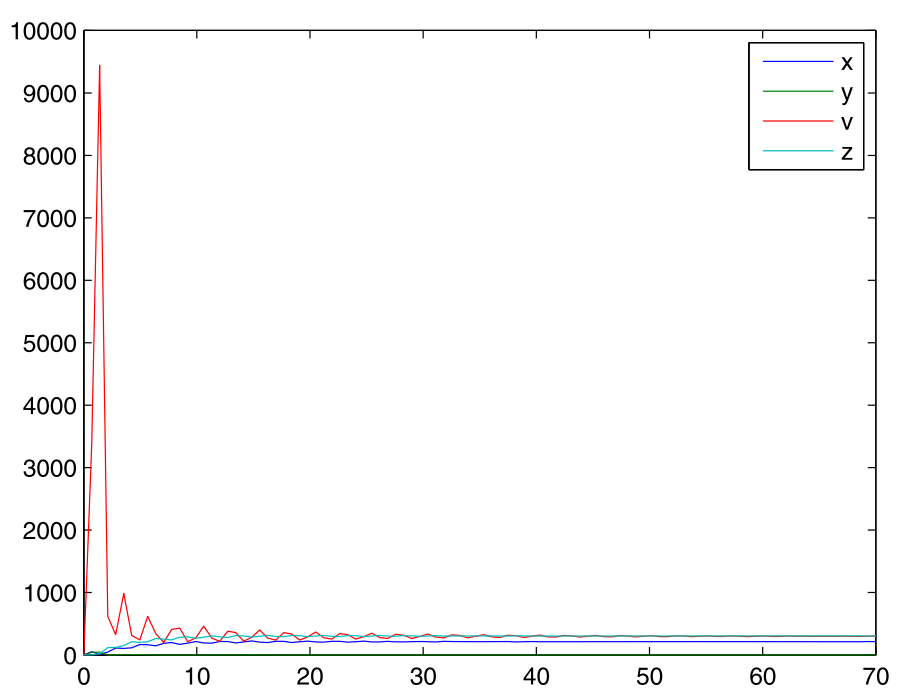

Figure 1 The plot shows the numerical solution of the system (1).

This indeed suggests that delay is a very important fact which should not be missed in HIV-1 modeling. Finally, through numerical simulations, it can be concluded that delays in the infection process and virus production period play an important role in the disease control.

\section{Competing interests}

The authors declare that they have no competing interests.

\section{Authors' contributions}

All authors contributed to the expression of the model, the discussion of results, and they wrote and approved the paper.

\section{Author details}

'Department of Mathematics, University of Malakand, Chakdara Dir (Lower), Khyber Pukhtoonkhwa, Pakistan.

${ }^{2}$ Department of Mathematics, Science College, King Saud University, Riyadh, Saudi Arabia.

\section{Acknowledgements}

We would like to thank the anonymous referees for their careful reading of the original manuscript and their many valuable comments and suggestions that greatly improved the presentation of this study. This work has been partially supported by King Saud University, Saudi Arabia.

Received: 2 November 2015 Accepted: 10 March 2016 Published online: 31 March 2016

\section{References}

1. Culshaw, RV, Ruan, S, Webb, G: A mathematical model of cell-to-cell spread of HIV-1 that includes a time delay. J. Math. Biol. 46(5), 425-444 (2003)

2. Culshaw, RV, Ruan, S: A delay-differential equation model of HIV-1 infection of CD4+ T-cells. Math. Biosci. 165(1), 27-39 (2000)

3. Nelson, PW, Murray, JD, Perelson, AS: A model of HIV-1 pathogenesis that includes an intracellular delay. Math. Biosci. 163(2), 201-215 (2000)

4. Zhu, H, Zou, X: Impact of delays in cell infection and virus production on HIV-1 dynamics. Math. Med. Biol. 25(2), 99-112 (2008)

5. Canabarro, AA: Periodic solutions and chaos in a non-linear model for the delayed cellular immune response. Physica A 342(1-2), 234-241 (2004)

6. Wang, K, Wang, W, Pang, H, Liu, X: Complex dynamic behavior in a viral model with delayed immune response. Physica D 226(2), 197-208 (2007)

7. Pawelek, KA, Liu, S, Pahlevani, F, Rong, L: A model of HIV-1 infection with two time delays, mathematical analysis and comparison with patient data. Math. Biosci. 235(1), 98-109 (2012)

8. Lashari, AA, Hattaf, K, Zaman, G: A delay differential equation model of a vector borne disease with direct transmission. Int. J. Ecol. Econ. Stat. 27, 25-35 (2012)

9. Zhu, H, Zou, X: Dynamics of a HIV-1 infection model with cell-mediated immune response and intracellular delay. Discrete Contin. Dyn. Syst., Ser. B 12(2), 511-524 (2009) 
10. Wang, X, Elaiw, A, Song, X: Global properties of a delayed HIV infection model with CTL immune response. Appl. Math. Comput. 218(18), 9405-9414 (2012)

11. Zhu, H, Luo, Y, Chen, M: Stability and Hopf bifurcation of a HIV infection model with CTL-response delay. Comput. Math. Appl. 62(8), 3091-3102 (2011)

12. Beretta, E, Kuang, Y: Geometric stability switch criteria in delay differential systems with delay dependent parameters SIAM J. Math. Anal. 33(5), 1144-1165 (2002)

13. Zaman, G, Kang, YH, Jung, IH: Optimal control in the SIR epidemic model with time delay. Biosystems $98,43-50$ (2009)

14. Zhang, T, Liu, J, Teng, Z: Stability of Hopf bifurcation of a delayed SIRS epidemic model with stage structure. Nonlinear Anal., Real World Appl. 11(1), 293-306 (2010)

15. Song, X, Zhou, X, Zhao, X: Properties of stability and Hopf bifurcation for a HIV infection model with time delay. Appl. Math. Model. 34(6), 1511-1523 (2010)

16. Miao, H, Abdurahman, X, Muhammadhaji, A: Global stability of HIV-1 infection model with two time delays. Abstr. Appl. Anal. 2013, Article ID 163484 (2013)

17. Wang, J, Wang, K, Jiang, Z: Dynamical behaviors of an HTLV-I infection model with intracellular delay and immune activation delay. Adv. Differ. Equ. 2015, 243 (2015)

18. Wang, J, Zhang, R, Kuniya, T: Mathematical analysis for an age-structured HIV infection model with saturation infection rate. Electron. J. Differ. Equ. 2015, 33 (2015)

19. Wang, J, Liu, S: The stability analysis of a general viral infection model with distributed delays and multi-staged infected progression. Commun. Nonlinear Sci. Numer. Simul. 20(1), 263-272 (2015)

20. Janeway, CA, Travers, P, Walport, M, Schlomchik, MJ: Immunobiology, 6th edn. Garland, New York (2005)

21. Kuang, Y: Delay Differential Equations with Applications in Population Dynamics. Academic Press, San Diego (1993)

22. LaSalle, J: The Stability of Dynamical Systems. SIAM, Philadelphia (1976)

23. Gantmacher, F: The Theory of Matrices, vol. 2. Chelsea, New York (1959)

\section{Submit your manuscript to a SpringerOpen ${ }^{\ominus}$ journal and benefit from:}

- Convenient online submission

- Rigorous peer review

- Immediate publication on acceptance

- Open access: articles freely available online

- High visibility within the field

Retaining the copyright to your article 